\title{
A Low Order Nonconforming Mixed Finite Element Scheme for Nonlinear Integro differential Equations of Pseudo-hyperbolic Type
}

\author{
Xianzhi Li, Kaiguang Zhang, Hongling Meng \\ Mathematics College, Zhengzhou normal University, Zhengzhou, china
}

\begin{abstract}
In this paper, a low order triangular nonconforming mixed finite element $\left(P_{1}+P_{0} \times P_{0}\right)$ scheme was studied for the nonlinear integro-differential equations of pseudo-hyperbolic type. By utilizing the properties of the interpolation, mean-value and derivative delivery techniques, the corresponding convergence analysis, the optimal error estimates of the original variable in -norm and intermediate variable $P$ in $L^{2}$-norm are obtained.
\end{abstract}

Keywords-nonlinear pseudo-hyperbolic integro-differential equation; triangular nonconforming finite element; new mixed finite element scheme; optimal error estimate.

\section{INTRODUCTION}

In recent years, along with the widely application in viscoelastic mechanics, nuclear reaction kinetics and biomechanics, the research of the integro-differential equations with pseudo-hyperbolic type attracts mach attention. We consider the following integro-differential equations with pseudo-hyperbolic type on a convex bounded region $\Omega$ with continuous boundary $\partial \Omega$,

$$
\left\{\begin{array}{l}
u_{t t}-\nabla \cdot\left(\nabla u_{t}+b(X, t) \nabla u+\int_{0}^{t} c(X, t, s) \nabla u(X, s) d s\right)=f(u, X, t),(X, t) \in \Omega \times J \\
u(X, t)=0,(X, t) \in \partial \Omega \times \bar{J} \\
u(X, 0)=u_{0}(X), u_{t}(X, 0)=u_{1}(X), X \in \Omega
\end{array}\right.
$$

Where $\Omega \subset R^{2}, J=(0, T]$, for Arbitrarily $T, T \in(0, \infty)$. The functions $b(X, t)$ and $c(X, t, s)$ respectively satisfy $0<b_{0} \leq b(X, t) \leq b_{1}<\infty, \quad 0<c_{0} \leq c(X, t, s) \leq c_{1}<\infty$, where $b_{0}, \quad b_{1}, c_{0}$ and $c_{1}$ are constants, $b, c, f, u_{0}$ and $u_{1}$ are known smooth functions. $f(u, X, t)$ is a nonlinear function with Lipschitz continuation about $u$. [1] gives a significant Sobolev-Volterra projection of equation (1), proves its existence and uniqueness as well as error estimate. [2] uses the method of H1-Galerkin mixed finite element discuss the existence and uniqueness of finite element solution, as well as its error estimate, proves the feasibility by numerical computation. [3] uses the method of mixed finite element give the semi-discretized optimal error estimate on the integro-differential equations of pseudo-hyperbolic Type. [4] uses the method of splitting positive mixed finite element give the semi-discretized and discretized optimal error estimate.

When using finite element to solve some problems, the critical function of original variation requires too high smoothness, making the structure of conforming finite element space requiring more degree of freedom. [5-8] using the intermediate variables to decrease the smooth degree of finite element space in order to solve these problems, but the two approximation space used by these methods need satify $B-B$ needs satisfaction needs satisfaction condition. For second order elliptic problem, elliptic problem $[9,10]$ propose a new scheme which means when the two finite element spaces satify a simple inclusion relation, $B-B$ needs satisfaction needs satisfaction condition is certainly true. $[11,12]$ using the high precision technique, for second order elliptic problem and linear elastic problem, study the overconvergence property on $\left(Q_{11}+Q_{01} \times Q_{10}\right)$ conforming finite element space, these methods is applied to Sobolev equation in [13]. Using nonconforming linear triangle element Crouzeix-Raviart, [14] applies these methods to hyperbolic type integro-differential equation to obtain optimal error estimate. Using nonconforming $E Q_{1}^{\text {rot }}$ element, [15] applies these methods to parabolic type equation to obtain overconvergence property and extrapolation. Using triangle conforming element, [16] applies these methods to parabolic type integro-differential equation to obtain overapproximatiom and overconvergence property.

This paper applies the scheme in [17] to formulation(1), by using the properties of the interpolation, mean-value and derivative delivery techniques, to analyze the convergence property, the optimal error estimates of the original variable $u$ in $H^{1}$-norm and intermediate variable $P$ in $L^{2}$-norm are obtained.

\section{MIXED FINITE ELEMENT FORMULATION}

Assume $\Gamma_{h}$ is a regular triangular subdivision to domain $\Omega$, for arbitrary $K \in \Gamma_{h}$, the coordinates of three vertices are $a_{i}\left(x_{i}, y_{i}\right), i=1,2,3$ respectively, the three edges are $l_{1}=\overline{a_{2} a_{3}}$, $l_{2}=\overline{a_{3} a_{1}}, l_{3}=\overline{a_{1} a_{2}}, h=\max _{k \in \Gamma_{k}} h_{K}$ respectively, where $h_{K}$ is the longest diameter of the unit $K$. Define the finite element spaces $V^{h}$ and $M^{h}$ respectively are $V^{h}=\left\{v^{h} ;\left.v^{h}\right|_{K} \in P_{1}(K), \int_{F}\left[v^{h}\right] d s=0, F \subset K, \quad \forall K \in \Gamma_{h}\right\} \quad, \quad$ and $M^{h}=\left\{P^{h} ;\left.P^{h}\right|_{K} \in P_{0}(K) \times P_{0}(K), \forall K \in \Gamma_{h}\right\}$, where $P_{1}(K)=\operatorname{span}\{1, x, y\}$, $P_{0}(K) \times P_{0}(K)=\operatorname{span}\{1\} \times \operatorname{span}\{1\}, \quad{ }^{\left[{ }^{h}\right]}$ is a jump value of edge $F$. Define the interpolating operators $I_{h}^{1}$ and $I_{h}^{2}$ respectively are

$$
\begin{gathered}
I_{h}^{1}: u \in H^{1}(\Omega) \rightarrow I_{h}^{1} u \in V^{h}, \int_{l_{i}}\left(u-I_{h}^{1} u\right) d s=0, i=1,2,3 \\
\text { and } I_{h}^{2}: P=\left(p_{1}, p_{2}\right) \in\left(L^{2}(\Omega)\right)^{2} \rightarrow I_{h}^{2} P \in M^{h}, \int_{K}\left(P-I_{h}^{2} P\right) d x d y=0 .
\end{gathered}
$$


It is obviously ${ }^{\mathrm{P} \bullet \mathrm{P}_{1, h}}$ is the module on $V^{h}$, and $\mathrm{P} \bullet \mathrm{P}_{1, h}=\left(\sum_{K \in \Gamma_{h}}|\bullet|_{1, K}^{2}\right)^{\frac{1}{2}}$, if $u \in H^{1}(\Omega)$, for arbitrary $\phi^{h} \in M^{h}$, we have

$$
\sum_{K \in \Gamma_{h}} \int_{K} \nabla\left(u-I_{h}^{1} u\right) \phi^{h} d x d y=O, \forall K \in \Gamma_{h} .
$$

According to $[19,20]$, for arbitrary $P \in\left(H^{1}(\Omega)\right)^{2}$ and $v^{v^{h} \in V^{h}}$, the following inequality is true

$$
\sum_{K \in \Gamma_{h}} \int_{\sigma K} P \cdot n v^{h} d s \leq c h|P| \mathrm{P} v^{h} \mathrm{P}_{1, h}, \forall K \in \Gamma_{h}
$$

where ${ }^{c}$ is a positive constant, and independent of $h$.

According to [2], the following inequality is true

$$
\int_{0}^{t} \int_{0}^{\tau}|\psi(s)|^{2} d s d \tau \leq c \int_{0}^{t}|\psi(s)|^{2} d s
$$

where ${ }^{\varphi}$ is an integrable function on ${ }^{[0, t]}$, for arbitrary $t \in[0, T]$.

For constructing the mixed finite element scheme of problem(1), we introduce the adjoint vectors function of $u$, $P=-\nabla u_{t}-b \nabla u-\int_{0}^{t} c(X, t, s) \nabla u(X, s) d s$, and then the problem could rewrite as the following first order system,

$$
\begin{cases}u_{t t}+\nabla \cdot P=f(u, X, t), & (X, t) \in \Omega \times J, \\ P+\nabla u_{t}+b \nabla u+\int_{0}^{t} c(X, t, s) \nabla u(X, s) d s=0, & (X, t) \in \Omega \times J, \\ u(X, t)=0, & (X, t) \in \partial \Omega \times \bar{J}, \\ u(X, 0)=u_{0}, u_{t}(X, 0)=u_{1}, & X \in \Omega .\end{cases}
$$

Its weekly formulation is extracting $\{u, P\}:[0, T] \rightarrow V \times M$ to satisfy

$$
\begin{cases}\left(u_{t}, v\right)-(P, \nabla v)=(f(u, X, t), v), & \forall v \in V, t \in J, \\ (P, q)+\left(\nabla u_{t}, q\right)+(b \nabla u, q)+\left(\int_{0}^{t} c(X, t, s) \nabla u(X, s) d s, q\right)=0, & \forall q \in M, t \in J, \\ u(X, 0)=u_{0}, u_{t}(X, 0)=u_{1}, & X \in \Omega .\end{cases}
$$

where $V=H_{0}^{1}(\Omega), M=\left(L^{2}(\Omega)\right)^{2}$.

The corresponding finite element approximation is extracting $\left\{u^{h}, P^{h}\right\}:[0, T] \rightarrow V^{h} \times M^{h}$ to satisfy

$$
\begin{cases}\left(u_{t}^{h}, v^{h}\right)-\left(P^{h}, \nabla v^{h}\right)=\left(f\left(u^{h}, X, t\right), v^{h}\right), & \forall v^{h} \in V^{h}, t \in J \\ \left(P^{h}, q^{h}\right)+\left(\nabla u_{t}^{h}, q^{h}\right)+\left(b \nabla u^{h}, q^{h}\right)+\left(\int_{0}^{t} c(X, t, s) \nabla u^{h}(X, s) d s, q^{h}\right)=0, & \forall q^{h} \in M^{h}, t \in J \\ u^{h}(X, 0)=I_{h}^{1} u_{0}, u_{t}^{h}(X, 0)=I_{h}^{1} u_{1}, & X \in \Omega\end{cases}
$$

\section{ERROR ANALYSIS}

Let $\quad u-u^{h}=u-I_{h}^{1} u+I_{h}^{1} u-u^{h}=\xi+\eta, \quad P-P^{h}=P-I_{h}^{2} P+I_{h}^{2} P-P^{h}=\rho+\theta$.

Theorem 1 assume ${ }^{(u, P)}$ and $\left(u^{h}, P^{h}\right)$ respectively are the solutions of the problem (6) and the problem (7), as $u, u_{t} \in H^{2}(\Omega), \quad P, P_{t} \in\left(H^{1}(\Omega)\right)^{2}, \quad u_{t t} \in H^{1}(\Omega) \quad$, we have

$$
\begin{aligned}
& \mathrm{P} u^{h}-u \mathrm{P}_{1, h} \leq \operatorname{ch}\left(|u|_{2}+\left(|P|_{1}^{2}+\int_{0}^{t}\left(|u|_{2}^{2}+\left|u_{t t}\right|_{1}^{2}+\left|P_{t}\right|_{1}^{2}\right) d s\right)^{\frac{1}{2}}\right), \\
& \mathrm{P} p^{h}-p \mathrm{P}_{L^{2}\left(0, T L^{2}\right)} \leq \operatorname{ch}\left(\int_{0}^{t}|P|_{1} d s+\left(|P|_{1}^{2}+\int_{0}^{t}\left(|u|_{2}^{2}+\left|u_{t t}\right|_{1}^{2}+\left|P_{t}\right|_{1}^{2}+|P|_{1}^{2}\right) d s\right)^{\frac{1}{2}}\right)
\end{aligned}
$$

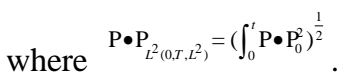

Proof For the first equation and the second equation in (5) are acted by $v^{h}\left(v^{h} \in V^{h}\right)$ and $q^{h}\left(q^{h} \in M^{h}\right)$ on the two sides respectively, using Green formulation, we have

$$
\left\{\begin{array}{c}
\left(u_{t t}, v^{h}\right)-\left(P, \nabla v^{h}\right)+\sum_{K} \int_{\partial K} P \cdot n v^{h} d s=\left(f(u, X, t), v^{h}\right), \forall v^{h} \in V^{h} \\
\left(P, q^{h}\right)+\left(\nabla u_{t}, q^{h}\right)+\left(b \nabla u, q^{h}\right)+\left(\int_{0}^{t} c(x, t, s) \nabla u(x, s) d s, q^{h}\right)=0, \forall q^{h} \in M^{h}
\end{array}\right.
$$

According to (7), we have the following error equation

$$
\left\{\begin{array}{c}
\left(\eta_{t t}, v^{h}\right)-\left(\theta, \nabla v^{h}\right)=-\left(\xi_{t t}, v^{h}\right)+\left(\rho, \nabla v^{h}\right)-\sum_{K} \int_{\partial K} P \cdot n v^{h} d s+\left(f(u, X, t)-f\left(u^{h}, X, t\right), v^{h}\right), \forall v^{h} \in V^{h} \\
\left(\theta, q^{h}\right)+\left(\nabla \eta_{t}, q^{h}\right)+\left(b \nabla \eta, q^{h}\right)+\left(\int_{0}^{t} c \nabla \eta d s, q^{h}\right)=-\left(\rho, q^{h}\right)-\left(\nabla \xi_{t}, q^{h}\right)-\left(b \nabla \xi, q^{h}\right)-\left(\int_{0}^{t} c \nabla \xi d s, q^{h}\right), \forall q^{h} \in M^{h}
\end{array}\right.
$$

Taking note of $\nabla v^{h}$ and $q^{h}$ are constants in every unit, $\left(\nabla v^{h}, \rho\right)=0,\left(\rho, q^{h}\right)=0,\left(\nabla \xi, q^{h}\right)=0$, and hence, the error equation could rewrite as

$\left\{\begin{array}{l}\left(\eta_{t t}, v^{h}\right)-\left(\theta, \nabla v^{h}\right)=-\left(\xi_{u}, v^{h}\right)-\sum_{K} \int_{\partial K} P \cdot n v^{h} d s+\left(f(u, X, t)-f\left(u^{h}, X, t\right), v^{h}\right), \forall v^{h} \in V \\ \left(\theta, q^{h}\right)+\left(\nabla \eta_{t}, q^{h}\right)+\left(b \nabla \eta, q^{h}\right)+\left(\int_{0}^{t} c \nabla \eta d s, q^{h}\right)=-\left(b \nabla \xi, q^{h}\right)-\left(\int_{0}^{t} c \nabla \xi d s, q^{h}\right), \forall q^{h} \in M^{h}\end{array}\right.$

Let $q^{h}=\nabla \eta$, the second equation in (12) becomes $\left(\nabla \eta_{t}, \nabla \eta\right)+(b \nabla \eta, \nabla \eta)+\left(\int_{0}^{t} \nabla \nabla \eta d s, \nabla \eta\right)=-(b \nabla \xi, \nabla \eta)-\left(\int_{0}^{t} c \nabla \xi d s, \nabla \eta\right)-(\theta, \nabla \eta)$ obviously, $\left(\nabla \eta_{t}, \nabla \eta\right)+(b \nabla \eta, \nabla \eta)+\left(\int_{0}^{t} c \nabla \eta d s, \nabla \eta\right)=\frac{1}{2} \frac{d}{d t} \mathrm{P} \eta \mathrm{P}_{1, h}^{2}+b_{0} \mathrm{P} \eta \mathrm{P}_{1, h}^{2}+\int_{0}^{t} c_{0} \mathrm{P} \eta \mathrm{P}_{1, h}^{2} d s$

For estimating the every term in the right set of (13), for arbitrary $\varphi(X) \in W^{1,0}(\Omega)$, define its mean in the unit $K$ as $\left.\bar{\varphi}(X)\right|_{K}=\frac{1}{|K|} \int_{K} \varphi(X) d X$, and then we have $|\varphi-\varphi|_{K} \leq \operatorname{ch} \mathrm{P} \varphi \mathrm{P}_{1, \alpha, K}$, using the mean technique and Young inequality, be aware of $(\bar{b} \nabla \xi, \nabla \eta)=0,(\bar{c} \nabla \xi, \nabla \eta)=0$,we have

$$
|(b \nabla \xi, \nabla \eta)|=\left|\sum_{K}((b-\bar{b}) \nabla \xi, \nabla \eta)_{K}\right| \leq c h^{4}|u|_{2}^{2}+\varepsilon \mathrm{P} \eta \mathrm{P}_{1, h}^{2},
$$

$$
\left|\left(\int_{0}^{t} c \nabla \xi d s, \nabla \eta\right)\right|=\left|\sum_{K}\left(\int_{0}^{t}(c-\bar{c}) \nabla \xi d s, \nabla \eta\right)_{K}\right| \leq c h^{4} \int_{0}^{t}|u|_{2}^{2} d s+\varepsilon \mathrm{P} \eta \mathrm{P}_{1, h}^{2}
$$

On the basis of Schwartz inequality, we obtain $|(\theta, \nabla \eta)| \leq \mathrm{P} \theta \mathrm{P}_{0} \mathrm{P} \nabla \eta \mathrm{P}_{0} \leq c \mathrm{P} \theta \mathrm{P}_{0}^{2}+\varepsilon \mathrm{P} \eta \mathrm{P}_{1, h}^{2}$

As $\varepsilon \rightarrow 0$, it becomes

$$
\frac{1}{2} \frac{d}{d t} \mathrm{P} \eta \mathrm{P}_{1, h}^{2} \leq c h^{4}\left(|u|_{2}^{2}+\int_{0}^{t}|u|_{2}^{2}\right)+c \mathrm{P} \theta \mathrm{P}_{0}^{2}
$$

Integrate two side of (14), and pay attention to $\eta(X, 0)=0$ we have

$$
\mathrm{P} \eta \mathrm{P}_{1, h}^{2} \leq c h^{4} \int_{0}^{t}|u|_{2}^{2}+c \int_{0}^{t} \mathrm{P} \theta \mathrm{P}_{0}^{2} d s
$$

In the first two equation of (12), respectively set $v^{h}=\eta_{t}$ and $q^{h}=\theta$, then add them, we obtain $\left(\eta_{t}, \eta_{t}\right)+(\theta, \theta)=\left(f(u, X, t)-f\left(u^{h}, X, t\right), \eta_{t}\right)-(b \nabla \eta, \theta)-\left(\int_{0}^{t} c \nabla \eta d s, \theta\right)-(b \nabla \xi, \theta)$

$$
-\left(\int_{0}^{t} c \nabla \xi d s, q^{h}\right)-\left(\xi_{t}, \eta_{t}\right)+\sum_{K} \int_{\partial K} P_{t} \cdot n \eta d s-\frac{d}{d t} \sum_{K} \int_{\partial K} P \cdot n \eta d s=\sum_{i=1}^{8} A_{i}
$$

Using the properties of $f$ and Young inequality, the estimate of $A_{1}$ is

$\left|\mathrm{A}_{1}\right| \leq c \mathrm{P} u-u^{h} \mathrm{P}_{0} \mathrm{P} \eta_{t} \mathrm{P}_{0} \leq c\left(\mathrm{P} u-I_{h}^{1} u \mathrm{P}_{0}+\mathrm{P} \eta \mathrm{P}_{0}\right)^{2}+\varepsilon_{1} \mathrm{P} \eta_{t} \mathrm{P}_{0}^{2} \leq c h^{2}|u|_{1}^{2}+c \mathrm{P} \eta \mathrm{P}_{0}^{2}+\varepsilon_{1} \mathrm{P} \eta_{t} \mathrm{P}_{0}^{2}$

On the basis of interpolation theory, Schwartz inequality and Young inequality, we obtain 
$\left|A_{2}\right| \leq c \mathrm{P} \eta \mathrm{P}_{1, h}^{2}+\varepsilon_{2} \mathrm{P} \theta \mathrm{P}_{0}^{2}, \quad\left|A_{3}\right| \leq c \int_{0}^{t} \mathrm{P} \eta \mathrm{P}_{1, h}^{2} d s+\varepsilon_{3} \mathrm{P} \theta \mathrm{P}_{0}^{2},\left|A_{6}\right| \leq c h^{2}\left|u_{t t}\right|_{1}^{2}+\varepsilon_{6} \mathrm{P} \eta_{t} \mathrm{P}_{0}^{2}$. have

For $(\bar{b} \nabla \xi, \theta)=0,(\bar{c} \nabla \xi, \theta)=0$, similar to(14) and (15), we

$$
\begin{array}{r}
\left|A_{4}\right|=\left|\sum_{K}((b-\bar{b}) \nabla \xi, \theta)_{K}\right| \leq c h^{4}|u|_{2}^{2}+\varepsilon_{4} \mathrm{P} \theta \mathrm{P}_{0}^{2} \\
\left|A_{5}\right|=\left|\sum_{K}\left(\int_{0}^{t}(c-\bar{c}) \nabla \xi d s, \theta\right)_{K}\right| \leq c h^{4} \int_{0}^{t}|u|_{2}^{2}+\varepsilon_{5} \mathrm{P} \theta \mathrm{P}_{0}^{2}
\end{array}
$$

On the basis of (2), we have

$$
\left|\mathrm{A}_{,}\right| \leq c h\left|P_{t}\right|_{1} \mathrm{P} \eta \mathrm{P}_{1, h} \leq c h^{2}\left|P_{t}\right|_{1}^{2}+\varepsilon_{7} \mathrm{P} \eta \mathrm{P}_{1, h}^{2} .
$$

Put them into (19), and thus the formulation (19) becomes

$$
\begin{aligned}
& \frac{1}{2} \frac{d}{d t} \mathrm{P} \eta_{t} \mathrm{P}_{0}^{2}+\mathrm{P} \theta \mathrm{P}_{0}^{2} \leq c \mathrm{P} \eta \mathrm{P}_{1, h}^{2}+c \int_{0}^{t} \mathrm{P} \eta \mathrm{P}_{1, h}^{2} d s+c h^{2}\left(|u|_{2}^{2}+|u|_{1}^{2}+\left|u_{t}\right|_{1}^{2}+\left|P_{t}\right|_{1}^{2}+\int_{0}^{t}|u|_{2}^{2}\right) \\
& +c \mathrm{P} \eta \mathrm{P}_{0}^{2}+\varepsilon \mathrm{P} \eta_{t} \mathrm{P}_{0}^{2}-\frac{d}{d t} \sum_{K} \int_{\partial K} P \cdot n \eta d s .
\end{aligned}
$$

Integrate two side, and pay attention to $\eta(X, 0)=\eta_{t}(X, 0)=0$, $\mathrm{P} \eta \mathrm{P}_{0}^{2} \leq \int_{0}^{t} \mathrm{P} \eta_{t} \mathrm{P}_{0}^{2}$, we obtain

$\mathrm{P} \eta_{t} \mathrm{P}_{0}^{2}+\int_{0}^{t} \mathrm{P} \theta \mathrm{P}_{0}^{2} d s \leq c h^{2} \int_{0}^{t}\left(|u|_{2}^{2}+|u|_{1}^{2}+\left|u_{t}\right|_{1}^{2}+\left|P_{t}\right|_{1}^{2}\right) d s+c \int_{0}^{t} \mathrm{P} \eta_{t} \mathrm{P}_{0}^{2} d s$

$+c \int_{0}^{t} \mathrm{P} \eta \mathrm{P}_{1, h}^{2} d s+c h^{2}|P|_{1}^{2}+c \varepsilon \mathrm{P} \eta \mathrm{P}_{1, h}^{2}$

According to Gronwall lemma, we have

$\int_{0}^{t} \mathrm{P} \theta \mathrm{P}_{0}^{2} d s \leq c h^{2}\left(|P|_{1}^{2}+\int_{0}^{t}\left(|u|_{2}^{2}+|u|_{1}^{2}+\left|u_{t}\right|_{1}^{2}+\left|P_{t}\right|_{1}^{2}\right) d s\right)+c \int_{0}^{t} \mathrm{P} \eta \mathrm{P}_{1, h}^{2} d s+c \varepsilon \mathrm{P} \eta \mathrm{P}_{1, h}^{2}$

as $\varepsilon \rightarrow 0$, using Gronwall lemma , (15) and (17), we obtain

$$
\begin{array}{r}
\mathrm{P} \eta \mathrm{P}_{1, h}^{2} \leq c h^{2}\left(|P|_{1}^{2}+\int_{0}^{t}\left(|u|_{2}^{2}+|u|_{1}^{2}+\left|u_{t t}\right|_{1}^{2}+\left|P_{t}\right|_{1}^{2}\right) d s\right), \\
\mathrm{P} \theta \mathrm{P}_{L^{2}\left(0, T L^{2}\right)} \leq c h^{2}\left(|P|_{1}^{2}+\int_{0}^{t}\left(|u|_{2}^{2}+|u|_{1}^{2}+\left|u_{t t}\right|_{1}^{2}+\left|P_{t}\right|_{1}^{2}+|P|_{1}^{2}\right) d s\right)
\end{array}
$$

On the basis of triangle inequality and interpolation theory, we obtaion (8) and (9) are true.

\section{REFERENCES}

[1] Cui,X.. Sobolev-volterra projection and numerical analysis of finite element methods for integro-differential equations. Acta Mathematicae Applicatae Sinica, 23 (4), pp. 441-454, 2001.

[2] Liu,Y., Li, H., He,S.R.G.L.. Errors estimate of H1-galerkin mixed finite element methods for pseudo-hyperbolic partial integro-differential equation. Numerical Mathematics A Journal of Chinese Universities, 32(1), pp. 1-20,2010.

[3] Ji,Z.Y., Li,H., Liu,Y.. Error Estimates Using a Semi-discrete Mixed Element Method for Pseudo-hyperbolic Integro-differential Equations. Journal of Inner Mongolia University(Natural Science Edition), 43(2),pp. 170-176,2011.

[4] Yu,Y.H., Liu,Y.. A splitting positive definite mixed element method for pseudo-hyperbolic integro-differential equations. Journal of Inner Mongolia University (Natural Science Edition), 43(4),pp. 377-383,2012.
[5] Pani,A.K., Yuan,J.Y.. Mixed finite element methods for a strongly damped wave equation. Numer. Methods Partial Differ.Equ. 17,pp. 105-119,2010.

[6] Shi,D.Y., Wang,H.H.. A New H1-Galerkin Mixed Finite Element Method for the Hyperbolic Type Integro-differential Equation. Chinese Journal of Engineering Mathematics, 26(4) ,pp. 648-652,2009.

[7] Luo,Z.D.. The basis and application of Mixed finite element, Science Press, BJ(2006), in press.

[8] Yang Liu, Hong Li. A New Mixed Finite Element Method for Pseudo-Hyperbolic Equation. Mathematica Applicata, 23(1),pp. 150-157,2009.

[9] Shaochun Chen, Hongru Chun. New mixed element schemes for second order elliptic problem. Mathematica Numerica Sinica, 32(2),pp. 213-218,2010.

[10] Shi,F., Yu,J.P., Li,K.T.. A New Mixed Finite Element Scheme for Elliptic Equations. Chinese Journal of Engineering Mathematics, 28(2),pp. 231-236,2011.

[11] Shi,D.Y., Li,M.H.. High accuracy analysis of new schemes for second order elliptic problem for recurrent event data. Acta Mathematicae Applicatae Sinica, 37(1),pp. 45-58,2014.

[12] Shi,D.Y., Li,M.H.. Super convergence analysis of the stable conforming rectangular mixed finite elements for the linear elasticity problem. Journal of Computational Mathematics, 32(2) ,pp. 205-214,2014.

[13] Shi,Y.H.. High accuracy analysis of a nre mixed finite element method for sobolev equation. Journal of Systems Science and Mathematical Sciences, 34(4),pp. 452-463,2014.

[14] Wu,Z.Q.. A New Nonconforming Mixed Finite Element Formulation for Hyperbolic Type Integro-Differential Equations. Journal of Jiangxi Normal University(Natural Science Edition), 6(5) ,p p. 487-490,2012.

[15] Shi,D.Y.. Superconvergence and extrapolation analysis of a new nonconforming mixed finite element approximation for parabolic equation. Mathematica Numerica Sinica, 43(17),pp. 242-246,2013.

[16] Wang,J.B., Wu,Z.Q., Shi,D.Y.. Superconvergence Analysis of a New Lowest Order Nonconforming Mixed Finite Element Formulation. Mathematics in Practice and Theory, 42(17) ,pp. 242-246,2013.

[17] Wu,Z.Q., Shi,D.Y.. A new low order nonconforming mixed finite element formulation for integro-differential equations of parabolic type. Journal of Anhui University(Natural Science Edition), 36(6) ,pp. 1-4,2012.

[18] Ciarlet,P.G. The finite element method for elliptic problems. North-Holland: Amsterdam(1978), in press.

[19] Shi,D.Y., Xu,C.. Anisotropic nonconforming Crouzeix-Raviart type FEM for second-order elliptic problems. Applied Mathematics and Mechanics, 33(2),pp. 243-252,2012.

[20] Zhang,Y.D., Shi,D.Y.. Convergence analysis of a new nonconforming mixed finite element for parabolic equation on anisotropic mesh. Mathematica Numerica Sinica, 35(2),pp. $171-180,2013$. 